\title{
Oxymatrine attenuates oxidized low-density lipoprotein-induced HUVEC injury by inhibiting NLRP3 inflammasome-mediated pyroptosis via the activation of the SIRT1/Nrf2 signaling pathway
}

\author{
XIN JIN $^{1}$, WAN FU ${ }^{2}$, JIAXIU ZHOU ${ }^{3}$, NIANNIAN SHUAI ${ }^{3}$, YAN YANG ${ }^{3}$ and BO WANG ${ }^{3}$ \\ ${ }^{1}$ Department of Anesthesiology, Affiliated Nanhua Hospital, University of South China; \\ Departments of ${ }^{2}$ Neurology and ${ }^{3}$ Anesthesiology, The First Affiliated Hospital, University of South China, \\ Hengyang, Hunan 421001, P.R. China
}

Received October 28, 2020; Accepted April 13, 2021

DOI: $10.3892 / \mathrm{ijmm} .2021 .5020$

\begin{abstract}
Oxymatrine, a quinolizidine alkaloid isolated from the traditional Chinese herb Sophora flavescens Aiton, has been demonstrated to exert anti-inflammatory and atherosclerotic effects, but the molecular mechanism has yet to be elucidated. Accumulating evidence indicates an important role of NLR family pyrin domain containing 3 (NLRP3) inflammasome-mediated pyroptosis in the pathogenesis of atherosclerosis. The present study was undertaken to investigate whether oxymatrine attenuates oxidized low-density lipoprotein (ox-LDL)-induced human umbilical vein endothelial cell (HUVEC) injury, an in vitro cell model of atherosclerosis, by inhibiting NLRP3 inflammasome-mediated pyroptosis, and elucidate the role of the sirtuin (SIRT)1/nuclear factor-erythroid 2-related factor 2 (Nrf2) signaling pathway in this process. Cell viability and cytotoxicity were detected by CCK- 8 assay and a lactate dehydrogenase (LDH) assay kit. Cell apoptosis was detected by flow cytometry. Reactive oxygen species (ROS) generation was detected using a ROS assay kit. The malondialdehyde (MDA) content, mitochondrial membrane potential (MMP) level, superoxide dismutase (SOD), catalase (CAT) and glutathione peroxidase (GSH-Px) activities were determined using commercial kits. The inflammatory cytokines levels were measured by ELISA and protein expression was monitored by western blot analysis. The results revealed that oxymatrine alleviated ox-LDL-induced cytotoxicity and apoptosis. Concurrently, oxymatrine inhibited ox-LDL-induced NLRP3 inflammasome-mediated pyroptosis
\end{abstract}

Correspondence to: Dr Bo Wang, Department of Anesthesiology, The First Affiliated Hospital, University of South China, 69 Chuanshan Road, Hengyang, Hunan 421001, P.R. China E-mail:wb621usc@126.com

Key words: oxymatrine, atherosclerosis, endothelial cell injury, NLRP3 inflammasome-mediated pyroptosis, sirtuin 1/nuclear factor-erythroid 2-related factor 2 signaling pathway in HUVECs, as evidenced by the significant decreases in the expression of NLRP3, apoptosis-associated speck-like protein containing a $\mathrm{C}$-terminal caspase recruitment domain (ASC), cleaved caspase-1, interleukin (IL)- $1 \beta$ and IL-18 in HUVECs. In addition, NLRP3 siRNA transfection efficiently suppressed ox-LDL-induced pyroptosis and HUVEC injury. Furthermore, oxymatrine promoted SIRT1/Nrf2 signaling pathway activation in HUVECs subjected to ox-LDL treatment, and SIRT1 deficiency induced by SIRT1 siRNA transfection abolished the protective effect of oxymatrine against ox-LDL-induced injury. SIRT1 siRNA also mitigated the oxymatrine-induced decreases in ROS generation and MDA content, and the increases in MMP as well as the activities of SOD, CAT and GSH-Px in HUVECs. Moreover, SIRT1 siRNA transfection blocked the inhibitory effect of oxymatrine on NLRP3 inflammasome-mediated pyroptosis in ox-LDL-treated HUVECs. Collectively, these results indicated that oxymatrine may attenuate ox-LDL-induced HUVEC injury by inhibiting NLRP 3 inflammasome-mediated pyroptosis via activating the SIRT1/Nrf2 signaling pathway.

\section{Introduction}

Atherosclerosis, a chronic inflammatory disease, is an important pathological process involved in peripheral artery disease, coronary artery disease and stroke, and has been attracting research interest during recent years $(1,2)$. A substantial number of studies have indicated that the NLR family pyrin domain containing 3 (NLRP3) inflammasome activation is implicated in the vascular inflammatory response driving the development and progression of atherosclerosis $(3,4)$. Upon activation, NLRP3 interacts with adapter apoptosis-associated speck-like protein containing a C-terminal caspase recruitment domain (ASC) to form the NLRP3 inflammasome, resulting in caspase-1 activation and the release of proinflammatory cytokines [i.e., interleukin (IL)-1 $\beta$ and IL-18], a process referred to as NLRP3 inflammasome activation-mediated pyroptosis (5-7), which has been reported as an important event in endothelial injury $(8,9)$ and the pathophysiological process of atherosclerosis $(10,11)$. Therefore, mitigation of NLRP3 inflammasome activation-mediated pyroptosis may 
be a promising therapeutic strategy for reversing endothelial injury in atherosclerosis.

Oxymatrine, a quinolizidine alkaloid isolated from the traditional Chinese herb Sophora flavescens Aiton, possesses multiple pharmacological properties, such as anti-inflammatory, antioxidant, antifibrotic and cardiovascular protective effects (12-14). To date, oxymatrine has been extensively discussed for its protective effects against heart injury induced by doxorubicin (14), chronic heart failure (15), aldosterone (16) and hypertension (17). Recent studies have claimed that oxymatrine inhibits homocysteine-induced human umbilical vein endothelial cell (HUVEC) apoptosis/death, attenuating endothelial cell dysfunction (a hallmark of atherosclerosis) $(18,19)$, thereby providing new insight into the role of oxymatrine in the prevention of atherosclerosis; however, the precise mechanisms have yet to be fully elucidated. Hence, the present study was undertaken to investigate the protective effect of oxymatrine against oxidized low-density lipoprotein (ox-LDL)-induced HUVEC injury, a typical in vitro model of atherosclerosis (20), and further demonstrated the role of NLRP3 inflammasome activation-mediated pyroptosis in this process.

Sirtuin (SIRT) 1, a member of the sirtuin family of $\mathrm{NAD}^{+}$-dependent deacetylases, has long been considered as an anti-atherosclerosis factor (21-23). SIRT1 can activate the nuclear factor-erythroid 2-related factor 2 (Nrf2) signaling pathway, and the activation of the Nrf2 pathway has been shown to attenuate endothelial cell injury, inhibit inflammation and exert anti-atherosclerotic effects $(24,25)$. Once activated, the Nrf2 signaling pathway can also inhibit NLRP3-mediated pyroptosis $(26,27)$. However, the exact effect of the SIRT1/Nrf2 signaling pathway on NLRP3 inflammasome activation-mediated pyroptosis in atherosclerosis has yet to be reported. Notably, it has been confirmed that oxymatrine can activate the SIRT1 signaling pathway $(28,29)$. Hence, the present study further explored the role of the SIRT1/Nrf2 signaling pathway in the protective effect of oxymatrine against ox-LDL injury and the inhibitory effect of oxymatrine on NLRP3 inflammasome activation-mediated pyroptosis in HUVECs.

The results of the present study may improve our understanding of the protective properties of oxymatrine agaisnt ox-LDL injury, indicating potential novel approaches to the treatment of atherosclerosis.

\section{Materials and methods}

Materials. ox-LDL, 2',7'-dichlorofluorescein diacetate (DCFH-DA) and dimethyl sulfoxide (DMSO) were purchased from Sigma-Aldrich; Merck KGaA. Oxymatrine (purity, >98\%) was obtained from Green Valley Pharmaceutical Co., Ltd. A stock suspension of oxymatrine $(50 \mathrm{mg} / \mathrm{ml})$ was prepared by dilution with PBS and stored at $4^{\circ} \mathrm{C}$. Dulbecco's modified Eagle's medium (DMEM; cat. no. SH30022.01B) was purchased from HyClone; Cytiva. Fetal bovine serum (FBS; cat. no. 16140071), doxorubicin and MTT assay kit (cat.no. V13154) were purchased from Gibco; Thermo Fisher Scientific, Inc. Annexin V-FITC/PI Apoptosis Detection kit was obtained from Shanghai Yisheng Technology Co., Ltd. (cat. no. 40302ES50). TNF- $\alpha$ assay kit (cat. no. H052), IL-1 $\beta$ assay kit (cat. no. H002), IL-6 assay kit (cat. no. H007) and IL-10 assay kit (cat. no. H009) were obtained from Nanjing Jiangcheng Bioengineering Institute. Anti-Bax (product no. 2772), anti-Bcl-2 (product no. 15071), anti-NLRP3 (product no. 13158), anti-cleaved caspase-1 (product no. 89332), anti-caspase-1 (product no. 24232) anti-IL-1 $\beta$ (product no. 12703), anti-IL-18 (product no. 54943), anti-SIRT1 (product no. 8469), anti-Nrf2 (product no. 12721), anti-heme oxygenase (HO)-1 (product no. 43966), anti-cleaved caspase-3 (product no. 9661), anti-caspase-3 (product no. 9662), anti-GAPDH (product no. 5174) and anti-histone H3 (product no. 4499) antibodies were purchased from Cell Signaling Technology, Inc. Small interfering (si)RNA for NLRP3 (siNLRP3; cat. no. sc-45469) and control siRNA-B (siNC; cat. no. sc-44230), siRNA for SIRT1 (siSIRT1; cat. no. sc-40986) and control siRNA-A (siCon; cat. no. sc-37007) were purchased from Santa Cruz Biotechnology, Inc.

HUVEC culture and treatment. HUVECs were purchased from the American Type Culture Collection and cultured in DMEM supplemented with $10 \%$ (v/v) FBS and $1 \%$ penicillin/streptomycin at $37^{\circ} \mathrm{C}$ in a humidified $5 \% \mathrm{CO}_{2}$ incubator. The culture medium was replaced every 1-2 days. HUVECs were treated with ox-LDL $(100 \mu \mathrm{g} / \mathrm{ml})$ for $24 \mathrm{~h}$ at $37^{\circ} \mathrm{C}$ to mimic atherosclerotic conditions in vitro (30). To detect the possible protective effects of oxymatrine against ox-LDL injury, HUVECs were pre-treated with oxymatrine $(2,4$ or $8 \mu \mathrm{M})$ for $1 \mathrm{~h}$ prior to ox-LDL $(100 \mu \mathrm{g} / \mathrm{ml})$ challenge for $24 \mathrm{~h}$ at $37^{\circ} \mathrm{C}$. To investigate the role of NLRP3 inflammasome activation-mediated pyroptosis in the protective effect of oxymatrine against ox-LDL-induced injury, HUVECs were transfected with siRNAs for NLRP3 (siNLRP3) followed by oxymatrine $(4 \mu \mathrm{M})$ for $1 \mathrm{~h}$ prior to ox-LDL $(100 \mu \mathrm{g} / \mathrm{ml})$ challenge for $24 \mathrm{~h}$. To demonstrate the function of the SIRT1/Nrf2 signaling pathway in the protective effect of oxymatrine against ox-LDL injury, HUVECs were transfected with siRNAs for SIRT1 (siSIRT1) followed by oxymatrine $(4 \mu \mathrm{M})$ for $1 \mathrm{~h}$ prior to ox-LDL $(100 \mu \mathrm{g} / \mathrm{ml})$ challenge for $24 \mathrm{~h}$. The dose of oxymatrine selected was according to our pilot study (unpublished data).

siRNA transfection. HUVECs were incubated in 6-well plates at $37^{\circ} \mathrm{C}$ overnight at a density of $1 \times 10^{5}$ cells/well, and then transfected with siRNAs for NLRP3 (siNLRP3, $50 \mathrm{nM}$ ) or siRNA for SIRT1 (siSIRT1, $50 \mathrm{nM}$ ), as well as a respective negative control (siNC, $50 \mathrm{nM}$ ) using Lipofectamine ${ }^{\circledR} 2000$ reagent (Invitrogen; Thermo Fisher Scientific, Inc.) following the manufacturer's protocol. The transfection sequences were as follows: siNLRP3 sense, 5'-GCUUCAGCCACA UGACUUUTT-3' and antisense, 5'-AAAGUCAUGUGG CUGAAGCTT-3'; siNC sense, 5'-UUCUCCGAACGUGUC ACGUTT-3' and antisense, 5'-ACGUGACACGUUCGGAGA ATT-3'; siSIRT1 sense, 5'-UUGGGAUUCACGCAUAGG AGCACUG-3' and antisense, 5'-CAGUGCUCCUAUGCG GAAUCCCAA-3'; and siRNA for control (siCon), sense 5'-UUCUCCGAACGUGUCACGUdTdT-3' and antisense 5'-ACGUGACACGUUCGGAGAAdTdT-3'. In brief, siNLRP3 $(50 \mathrm{nM})$, siSIRT1 $(50 \mathrm{nM})$, siNC $(50 \mathrm{nM})$ or siCon $(50 \mathrm{nM})$ was mixed with Lipofectamine ${ }^{\circledR} 2000$ transfection reagent, and the mixture was added to the cells and incubated for $6 \mathrm{~h}$ at 
$37^{\circ} \mathrm{C}$. Then the medium was replaced with complete medium and the cells were cultured for $24 \mathrm{~h}$, following which the cells were collected for subsequent experiments. The transfection efficiency of siNLRP3 and siSIRT1 in HUVECs was detected at $24 \mathrm{~h}$ after transfection by western blotting.

Cell viability assay. The viability of HUVECs was measured by Cell Counting Kit-8 (CCK-8) assay. In brief, HUVECs were grown in 96-well plates and treated as aforementioned. CCK-8 solution (10 $\mu \mathrm{l}$; cat. no. C0038; Beyotime Biotechnology) was added to each well and co-incubated for $3 \mathrm{~h}$ at $37^{\circ} \mathrm{C}$. The optical density (OD) at $450 \mathrm{~nm}$ was measured using a microplate reader (Thermo Fisher Scientific, Inc.).

Lactate dehydrogenase $(L D H)$ assay. The cell death was further detected using an LDH Cytotoxicity Assay kit (cat. no. C0017; Beyotime Biotechnology) according to the manufacturer's protocol. The LDH release was measured at $490 \mathrm{~nm}$ using a microplate reader (Bio-Rad Laboratories, Inc.).

Apoptosis assay. The apoptosis ratios of HUVECs were measured using Annexin V-FITC/PI Apoptosis Detection kit by flow cytometry according to manufacturer's instructions. Briefly, after treatment, HUVECs were collected, washed twice with PBS, and incubated with Annexin V-FITC (10 $\mu \mathrm{l})$ and PI $(5 \mu \mathrm{l})$ for $20 \mathrm{~min}$ at room temperature in the dark. The mixture was detected by flow cytometric analysis using a flow cytometer (FACSCalibur ${ }^{\mathrm{TM}}$; BD Biosciences), and the data were analyzed using FlowJo 7.6 software (Tree Star, Inc.).

Determination of reactive oxygen species (ROS) production. The production of intracellular ROS was detected by the DCFH-DA fluorescence probe according to the manufacturer's protocol. In brief, the treated HUVECs were harvested and washed twice with PBS, and then incubated with DCFH-DA $(10 \mu \mathrm{M})$ for $20 \mathrm{~min}$ in the dark at $37^{\circ} \mathrm{C}$. After washing again with PBS, images were captured under a fluorescence microscope (80i; Nikon Corporation). In addition, the mean fluorescence intensity of ROS was analyzed and averaged using flow cytometry (FACSCalibur ${ }^{\mathrm{TM}}$ ), and the data were analyzed by FlowJo 7.6 software (Tree Star, Inc.).

Determination of malondialdehyde (MDA) content, superoxide dismutase (SOD), catalase (CAT) and glutathione peroxidase (GSH-Px) activities. After treatment, HUVECs were harvested, washed with ice-cold PBS, and incubated with the lysis buffer provided in the assay kits for $30 \mathrm{~min}$ on ice. Subsequently, cell lysates were centrifuged at $10,000 \mathrm{x} g$ for $10 \mathrm{~min}$ at $4^{\circ} \mathrm{C}$, the supernatant was collected and the protein content was measured using bicinchoninic acid (BCA) protein assay kit (Beyotime Biotechnology). The MDA content (cat. no. A003-1-2), SOD (cat. no. A001-3-2), CAT (cat. no. A007-1-1) and GSH-Px (cat. no. A005-1-2) activities were determined by commercial kits from Nanjing Jiancheng Bioengineering Institute according to the manufacturers' protocol.

Measurement of mitochondrial membrane potential (MMP). The MMP was monitored using the JC-1 dye kit according to the manufacturers' protocol (cat. no. C2006; Beyotime
Biotechnology). Briefly, HUVECs were harvested, washed twice with PBS, and then incubated with JC-1 for $20 \mathrm{~min}$ at $37^{\circ} \mathrm{C}$ in the dark. The data were analyzed and averaged using flow cytometry (FACSCalibur ${ }^{\mathrm{TM}}$ ), and the data were analyzed by FlowJo 7.6 software (Tree Star, Inc.).

Western blot analysis. Total protein from HUVECs was isolated using RIPA lysis buffer (Beyotime Biotechnology) containing $1 \%(\mathrm{v} / \mathrm{v})$ protease inhibitor cocktail (Roche Diagnostics). The cytoplasmic and nuclear proteins of HUVECs were extracted with a cytoplasmic and nuclear extraction kit (cat. no. CW0199), according to the manufacturer's instructions (CWBIO). The protein concentration was measured with a BCA protein assay kit. Equal amounts of protein $(30 \mu \mathrm{g})$ were separated by $12 \%$ SDS-PAGE and then transferred onto PVDF membranes. After blocking with 5\% (w/v) skimmed dry milk powder dissolved in Tris-buffered saline containing $0.1 \%(\mathrm{v} / \mathrm{v})$ Tween-20 for $2 \mathrm{~h}$ at room temperature, the membranes were incubated with primary antibodies against $\operatorname{Bax}(1: 2,000), \mathrm{Bcl}-2$ $(1: 2,000)$, NLPR3 $(1: 1,000)$, ASC $(1: 1,000)$, cleaved caspase-1 $(1: 1,000)$, caspase-1 (1:2,000) IL-1 $\beta(1: 1,000)$, IL-18 $(1: 1,000)$, SIRT1 $(1: 2,000)$, Nrf2 (1:1,000), HO-1 (1:1,000), cleaved caspase-3 $(1: 2,000)$, caspase-3 $(1: 1,000)$, GAPDH $(1: 2,000)$ and histone $\mathrm{H} 3(1: 2,000)$ overnight at $4^{\circ} \mathrm{C}$. GAPDH was used as an internal control. Then, the membranes were incubated with HRP-conjugated secondary antibodies (1:5,000; product no. 7074; Cell Signaling Technology, Inc.) for $2 \mathrm{~h}$ at room temperature and the probed protein was visualized by Beyo ECL Plus (cat. no. P0018, Beyotime Biotechnology). The densitometric analysis was semi-quantified with ImageJ 1.43 software (National Institutes of Health).

Statistical analysis. Statistical analysis was performed by GraphPad Prism 5 (GraphPad Software, Inc.) using one-way analysis of variance (ANOVA) followed by Tukey's post hoc test. All the data are presented as the mean \pm SD. All experiments were repeated at least three times. $\mathrm{P}<0.05$ was considered to indicate a statistically significant difference.

\section{Results}

Oxymatrine alleviates ox-LDL-induced HUVEC injury. To assess the protective effect of oxymatrine against ox-LDL-induced injury in HUVECs, cells were pre-treated with varying concentrations of oxymatrine in the presence or absence of ox-LDL $(100 \mu \mathrm{g} / \mathrm{ml})$. The results revealed that oxymatrine $(2,4$ or $8 \mu \mathrm{M})$ significantly reversed the ox-LDL-induced decrease in cell viability (Fig. 1A). Treatment with oxymatrine alone $(2,4$ or $8 \mu \mathrm{M})$ did not affect cell viability compared with the control group. The results revealed that oxymatrine $(4 \mu \mathrm{M})$ obviously increased the viability of HUVECs treated with ox-LDL $(\mathrm{P}<0.01)$, which was selected as an optimal dose for interference with HUVECs treated with ox-LDL in a follow-up experiment. Subsequently, the result confirmed that oxymatrine $(4 \mu \mathrm{M})$ significantly attenuated the ox-LDL-induced increase in LDH release in HUVECs (Fig. 1B). Flow cytometric assay results further demonstrated that oxymatrine pretreatment also suppressed the ox-LDL-induced upregulation of the apoptotic percentage in HUVECs (Fig. 1C). Bcl-2 and Bax are important 


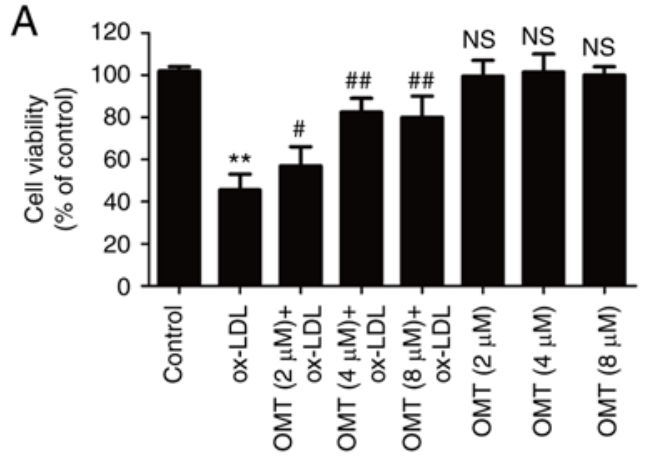

C
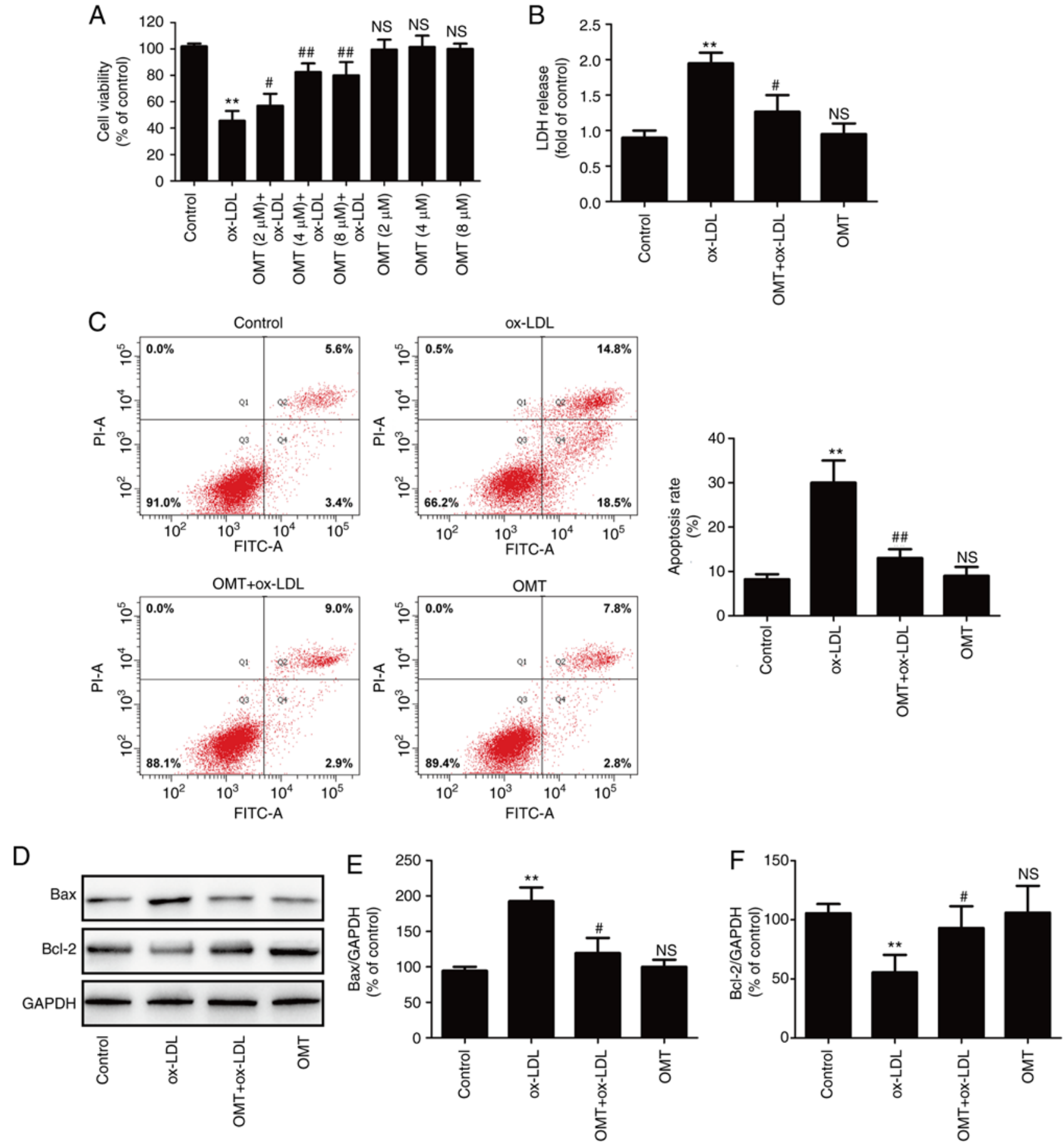

Figure 1. Effects of oxymatrine on cytotoxicity and apoptosis in ox-LDL-stimulated HUVECs. (A) HUVECs were pretreated with oxymatrine (2, 4 or $8 \mu \mathrm{M})$ for $2 \mathrm{~h}$ prior to treatment with ox-LDL $(100 \mu \mathrm{g} / \mathrm{ml})$ for $24 \mathrm{~h}$, followed by the evaluation of cell viability. Data are expressed as the mean $\pm \mathrm{SD}, \mathrm{n}=3$. ${ }^{* *} \mathrm{P}<0.01$ and ${ }^{\mathrm{NS}} \mathrm{P}>0.05$ vs. the control group; ${ }^{\#} \mathrm{P}<0.05$ and ${ }^{\# \#} \mathrm{P}<0.01$ vs. the ox-LDL group. Cells were pretreated with oxymatrine $(4 \mu \mathrm{M})$ for $2 \mathrm{~h}$ prior to treatment with ox-LDL (100 $\mu \mathrm{g} / \mathrm{ml}$ ) for $24 \mathrm{~h}$, followed by the determination of (B) LDH release, (C) apoptosis rate and (D) Bax and Bcl-2 expression. Quantitative analysis for (E) Bax/GAPDH and (F) Bcl-2/GAPDH. Data are expressed as the mean \pm SD, $n=3 .{ }^{* * *} \mathrm{P}<0.01$ and ${ }^{\mathrm{NS}} \mathrm{P}>0.05$ vs. the control group; ${ }^{\#} \mathrm{P}<0.05$ and ${ }^{\# \#} \mathrm{P}<0.01$ vs. the ox-LDL group. OMT, oxymatrine; ox-LDL, oxidized low-density lipoprotein; NS, no statistical significance; HUVECs, human umbilical vein endothelial cells; $\mathrm{LDH}$, lactate dehydrogenase.

members of the Bcl-2 family and they play a key role in the mitochondrial-mediated apoptotic pathway (31). The results of western blot analysis (Fig. 1D) further demonstrated that ox-LDL treatment resulted in an increase in the expression of the pro-apoptotic protein Bax (Fig. 1E) and a decrease in the expression of the anti-apoptotic protein Bcl-2 (Fig. 1F), while these effects were significantly blocked by oxymatrine.
These data indicated that oxymatrine protected HUVECs against ox-LDL-induced injury.

Oxymatrine inhibits NLRP3 inflammasome-mediated pyroptosis in HUVECs subjected to ox-LDL treatment. Several studies have revealed the important role of NLRP3 inflammasome in atherosclerosis $(4,32)$. The present 
study further investigated whether oxymatrine has the ability to reduce NLRP3 inflammasome activation during atherosclerosis. As shown in Fig. 2A, compared with the ox-LDL group, pretreatment with oxymatrine significantly decreased the expression of NLRP3 (Fig. 2B) and ASC (Fig. 2C) in HUVECs. The research confirmed that NLRP3 inflammasome activation leads to caspase-1 activation, thereby leading to maturation of effector pro-inflammatory cytokines, such as IL-1 $\beta$ and IL-18; thus, activated caspase-1 indicates the presence of pyroptosis (7). The results of western blot analysis (Fig. 2D) further demonstrated that ox-LDL resulted in the upregulation of the ratio of cleaved caspase-1/caspase-1 (Fig. 2E), IL-1 $\beta$ (Fig. 2F) and the expression of IL-18 (Fig. 2G) in HUVECs, while these effects were blocked by oxymatrine. These results indicated that oxymatrine attenuated NLRP3 inflammasome-mediated pyroptosis under conditions of ox-LDL stimulation.

Furthermore, to investigate the exact role of NLRP3 inflammasome-mediated pyroptosis in the protection of oxymatrine against ox-LDL injury, HUVECs were transfected with siNLRP3. The results demonstrated that siNLRP3 transfection successfully decreased the expression of NLRP3, ASC, cleaved caspase-1, IL-1 $\beta$ and IL-18 protein (Fig. $2 \mathrm{H}$ ) in HUVECs. Moreover, siNLRP3 transfection also mitigated the ox-LDL-induced decrease in cell viability (Fig. 2I), the increase in $\mathrm{LDH}$ release (Fig. 2J) and apoptosis rate (Fig. 2K) of HUVECs, indicating that inhibition of NLRP3 inflammasome activation-mediated pyroptosis attenuated ox-LDL-induced HUVEC injury. Collectively, these findings indicated that oxymatrine protected HUVECs against ox-LDL-induced injury through inhibition of NLRP3 inflammasome activation-mediated pyroptosis.

Oxymatrine promotes SIRT1/Nrf2 signaling pathway activation in HUVECs subjected to ox-LDL treatment. To further investigate the mechanism underlying oxymatrine-induced endothelial protection against ox-LDL injury, the effects of oxymatrine on the SIRT1/Nrf2 signaling pathway in HUVECs were investigated. As revealed in Fig. 3, ox-LDL treatment resulted in the downregulation of SIRT1 expression in HUVECs, while oxymatrine pretreatment led to the upregulation of SIRT expression (Fig. 3A and B) in ox-LDL-treated HUVECs. In addition, oxymatrine blocked the ox-LDL-induced increase in cytoplasmic Nrf2 (C-Nrf2) expression (Fig. 3A and $\mathrm{C}$ ) and decrease in nuclear Nrf2 (N-Nrf2) expression (Fig. 3E and F), indicating that oxymatrine may promote Nrf2 nuclear translocation. In addition, the ox-LDL-induced downregulation of the expression of the Nrf2-related antioxidant defense enzyme HO-1 was also reversed by oxymatrine (Fig. 3A and D). These results indicated that oxymatrine promoted the activation of the SIRT1/Nrf2 signaling pathway under conditions of ox-LDL stimulation in HUVECs.

Depletion of the SIRT1 signaling pathway blocks the protective effect of oxymatrine against ox-LDL-induced injury in HUVECs. To further explore the pathophysiological significance of oxymatrine-induced SIRT1/Nrf2 signaling pathway activation, SIRT1 knockdown was performed by transfection of siSIRT1 to further investigate the effect of oxymatrine against ox-LDL-induced injury. The results (Fig. 4A) demonstrated that siSIRT1 transfection markedly reduced the expression of SIRT1 compared with siCon transfection in HUVECs treated with oxymatrine and ox-LDL (Fig. 4B). siSIRT1 transfection also decreased the expression of HO-1 (Fig. 4C) and N-Nrf2 (Fig. 4D) in comparison with siCon transfection, indicating that siSIRT1 transfection successfully led to the deficiency of the SIRT1/Nrf2 signaling pathway. On this basis, the results further revealed that siSIRT transfection inhibited the oxymatrine-induced increase in cell viability (Fig. 4E) and the decrease in LDH release (Fig. 4F) in ox-LDL-treated HUVECs compared with siCon transfection. Furthermore, the inhibitory effect of oxymatrine on apoptosis rate was also alleviated by siSIRT1 transfection in comparison with siCon transfection (Fig. 4G). Caspase- 3 is the main executioner of apoptosis, and activated caspase-3 can induce the cells to undergo apoptosis (33). Oxymatrine reduced the ox-LDL-induced increase in the ratio of cleaved caspase-3/caspase-3, which was also reversed by siSIRT1 (Fig. 4H and I). Collectively, these data indicated that the SIRT1/Nrf2 signaling pathway may contribute to the protection of oxymatrine against ox-LDL injury in HUVECs.

Inhibition of the SIRTI/Nrf2 signaling pathway eliminates the oxymatrine-induced protective effect against oxidative stress in ox-LDL-stimulated HUVECs. The present study further investigated the role of the SIRT1/Nrf2 signaling pathway in the antioxidant activity of oxymatrine in atherosclerosis. As revealed in Fig. 5, oxymatrine pretreatment significantly reversed the ox-LDL-induced increase in endogenous ROS generation (Fig. 5A and B) and MDA content (Fig. 5C), a major marker of lipid peroxidation. However, these effects of oxymatrine were both blocked by siSIRT1 transfection. In addition, oxymatrine resulted in an increase in MMP in HUVECs subjected to ox-LDL, which was also reversed by siSIRT1 transfection (Fig. 5D). Excessive ROS production and/or failure of antioxidant defense systems lead to oxidative stress and cardiomyocyte injury (34). Hence, the effects of oxymatrine on antioxidant enzymes inducing SOD, CAT and GSH-Px activity and the role of the SIRT1/Nrf2 signaling pathway in this process were explored. The results demonstrated that oxymatrine pretreatment obviously attenuated the ox-LDL-induced decrease in SOD (Fig. 5E), GSH-Px (Fig. 5F) and CAT (Fig. 5G) activities in HUVECs. However, oxymatrine-induced increases in SOD (Fig. 5E), GSH-Px (Fig. 5F) and CAT (Fig. 5G) activities were blocked by siSIRT1 transfection. Collectively, these results indicated that the SIRT1/Nrf2 signaling pathway mediated the protective effects of oxymatrine against ox-LDL-induced oxidative damage in HUVECs.

Inhibition of the SIRT1/Nrf2 signaling pathway alleviates oxymatrine-reduced NLRP3 inflammasome-mediated pyroptosis in ox-LDL-stimulated HUVECs. Research has confirmed that SIRT1 signaling inhibits NLRP3 inflammasome activation in vascular endothelial cells (35). The present study was undertaken to further explore whether oxymatrine attenuates NLRP3 inflammasome activation-mediated pyroptosis in ox-LDL-stimulated HUVECs via promoting SIRT1/Nrf2 signaling. The findings (Fig. 6A) revealed that 

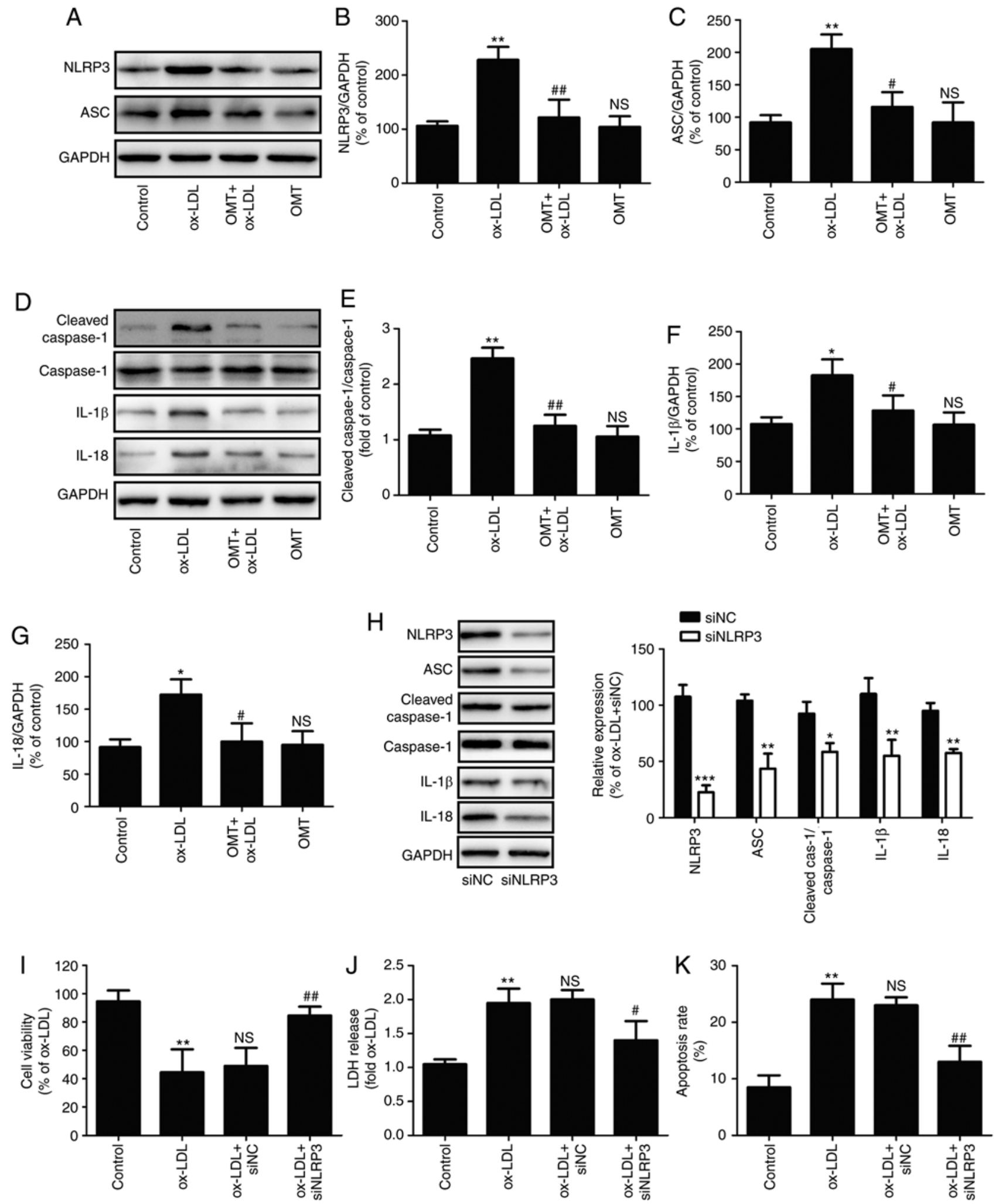

Figure 2. Effects of oxymatrine on NLRP3 inflammasome-mediated pyroptosis in ox-LDL-stimulated HUVECs. HUVECs were pretreated with oxymatrine $(4 \mu \mathrm{M})$ for $2 \mathrm{~h}$ prior to treatment with ox-LDL $(100 \mu \mathrm{g} / \mathrm{ml})$ for $24 \mathrm{~h}$. (A) Western blot analysis for NLRP3 and ASC protein expression. Quantitative analysis for (B) NLRP3/GAPDH and (C) ASC/GAPDH. (D) Western blot analysis for cleaved caspase-1, caspase-1, IL-1 $\beta$ and IL-18 protein expression levels. Quantitative analysis for (E) cleaved caspase-1/caspase-1, (F) IL-1 $/$ GAPDH and (G) IL-18/GAPDH. Data are expressed as the mean \pm SD, $n=3$. " $\mathrm{P}<0.05,{ }^{* *} \mathrm{P}<0.01$ and ${ }^{\mathrm{NS}} \mathrm{P}>0.05$ vs. the control group; ${ }^{\#} \mathrm{P}<0.05$ and ${ }^{\# \#} \mathrm{P}<0.01$ vs. the ox-LDL group. HUVECs were transfected with siNLRP 3 or siNC for $24 \mathrm{~h}$, followed by measurement of (H) NLRP3, ASC, cleaved caspase-1, caspase-1, IL-1 $\beta$ and IL-18 protein expression. Data are expressed as the mean $\pm \mathrm{SD}, \mathrm{n}=3$. ${ }^{*} \mathrm{P}<0.05,{ }^{* *} \mathrm{P}<0.01$, ${ }^{* * * *} \mathrm{P}<0.001$ vs. siNC group. Cells were transfected with siNLRP3 or siNC before treatment with oxymatrine $(4 \mu \mathrm{M})$ for $2 \mathrm{~h}$ prior to treatment with ox-LDL $(100 \mu \mathrm{g} / \mathrm{ml})$ for $24 \mathrm{~h}$. Determination of (I) cell viability, (J) LDH release and (K) apoptosis rate. Data are expressed as the mean $\pm \mathrm{SD}, \mathrm{n}=3 .{ }^{* *} \mathrm{P}<0.01 \mathrm{vs}$. the control group; ${ }^{~} \mathrm{P}<0.05$ and $^{\# \# /} \mathrm{P}<0.01$ vs. the ox-LDL + siNC group. OMT, oxymatrine; ox-LDL, oxidized low-density lipoprotein; NS, no statistical significance; siNLRP3, small interfering ribonucleic acid (siRNA) for NLRP3; siNC, siRNA for negative control; HUVECs, human umbilical vein endothelial cells; LDH, lactate dehydrogenase; NLRP3, NLR family pyrin domain containing 3; ASC, apoptosis-associated speck-like protein containing a C-terminal caspase recruitment domain; Cleaved cas-1, Cleaved caspase-1. 
A

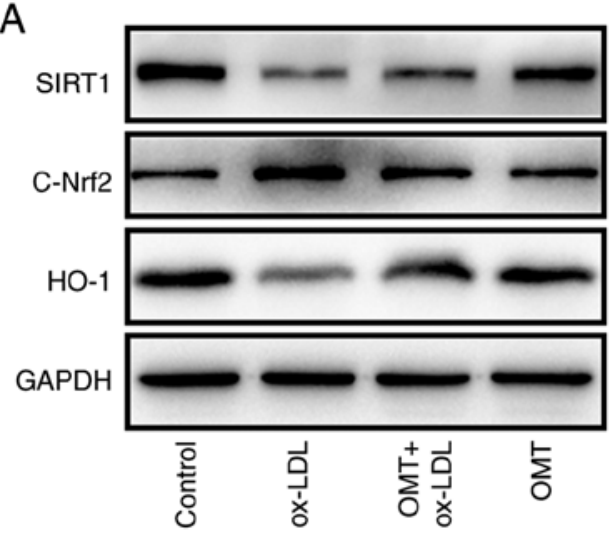

C
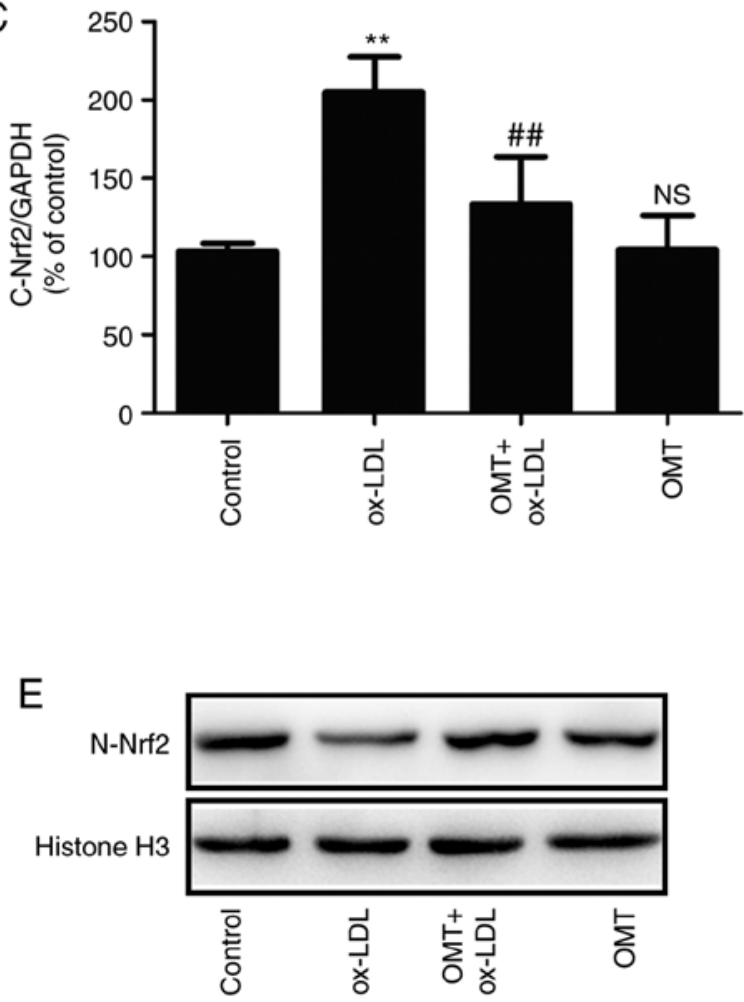
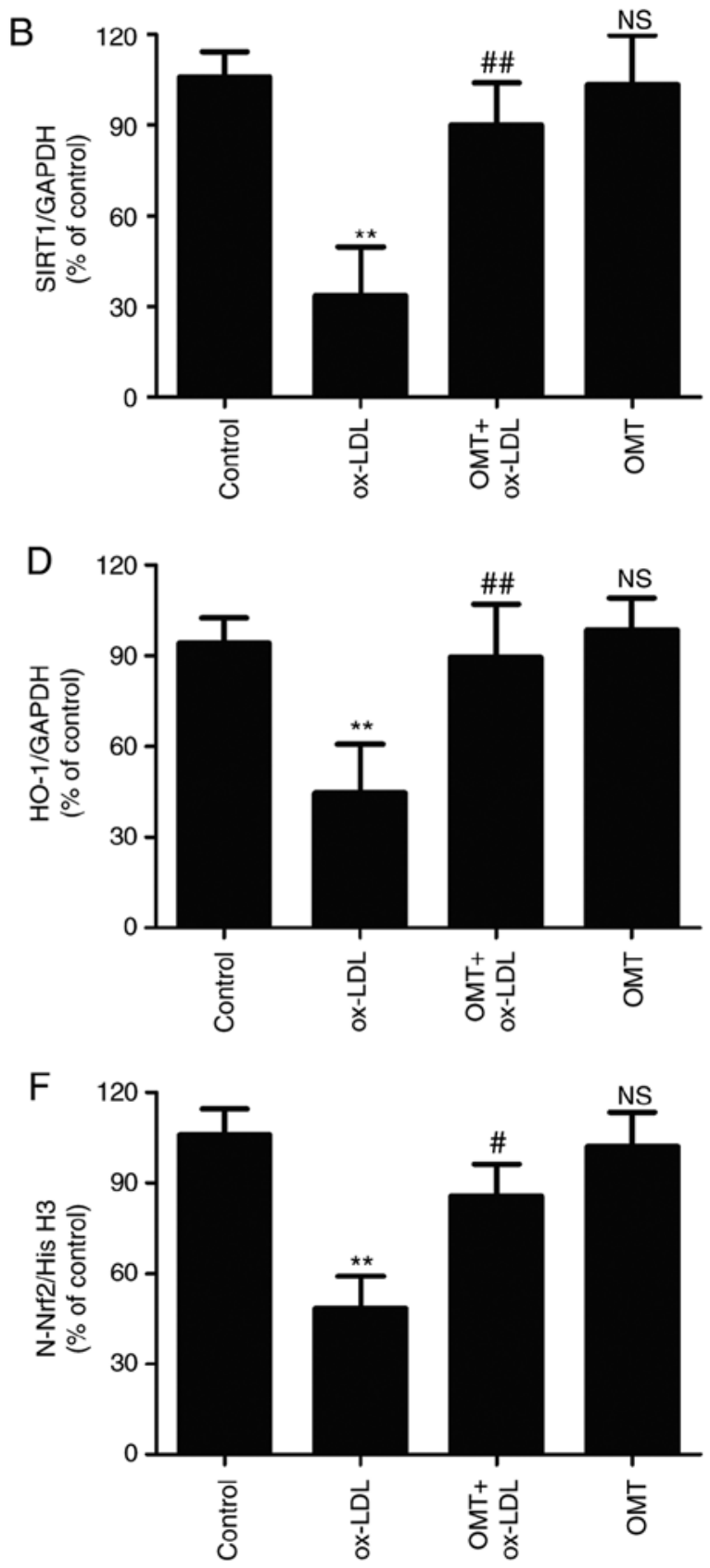

Figure 3. Effects of oxymatrine on the SIRT1/Nrf2 signaling pathway in ox-LDL-treated HUVECs. HUVECs were pretreated with oxymatrine (4 $\mu$ M) for $2 \mathrm{~h}$ prior to treatment with ox-LDL $(100 \mu \mathrm{g} / \mathrm{ml})$ for $24 \mathrm{~h}$. (A) Western blot analysis for SIRT1, C-Nrf2 and HO-1 protein expression. Quantitative analysis for (B) SIRT1, (C) C-Nrf2 and (D) HO-1 relative to GAPDH. (E) Western blot analysis for N-Nrf2 protein expression. (F) Quantitative analysis for Nrf2 relative to histone $\mathrm{H} 3$. Data are expressed as the mean $\pm \mathrm{SD}, \mathrm{n}=3 .{ }^{* *} \mathrm{P}<0.01$ and ${ }^{\mathrm{NS}} \mathrm{P}>0.05$ vs. the control group; ${ }^{\#} \mathrm{P}<0.05$ and ${ }^{\# \#} \mathrm{P}<0.01$ vs. the ox-LDL group. OMT, oxymatrine; ox-LDL, oxidized low-density lipoprotein; NS, no statistical significance; HUVECs, human umbilical vein endothelial cells; SIRT, sirtuin; Nrf2, nuclear factor-erythroid 2-related factor 2; C-Nrf2, cytoplasmic Nrf2; N-Nrf2, nuclear Nrf2; HO-1, heme oxygenase 1.

inactivation of the SIRT1/Nrf2 signaling pathway induced by siSIRT1 transfection weakened the oxymatrine-induced decrease in the expression of NLRP3 (Fig. 6B) and ASC (Fig. 6C) in ox-LDL-treated HUVECs. Transfection with siSIRT1 also inhibited the oxymatrine-induced decrease in the ratio of cleaved caspase-1/caspase-1 (Fig. 6D and E) and the expression of IL-1 $\beta$ (Fig. 6D and F) and IL-18 (Fig. 6D and G) in ox-LDL-treated HUVECs. These results indicated that the SIRT1/Nrf2 signaling pathway mediated the inhibitory effect of oxymatrine on NLRP3 inflammasome-mediated pyroptosis in ox-LDL-treated HUVECs.

\section{Discussion}

The present study investigated the protective effects of oxymatrine against ox-LDL-induced injury in HUVECs, and further demonstrated the underlying mechanisms, focusing on NLRP3 inflammasome-mediated pyroptosis and SIRT1/Nrf2 signaling. The results revealed that oxymatrine attenuated ox-LDL-induced cytotoxicity and apoptosis via inhibiting NLRP3 inflammasome-mediated pyroptosis. In addition, the SIRT1/Nrf2 signaling pathway mediated the protective effects of oxymatrine against ox-LDL injury and oxidative 
A
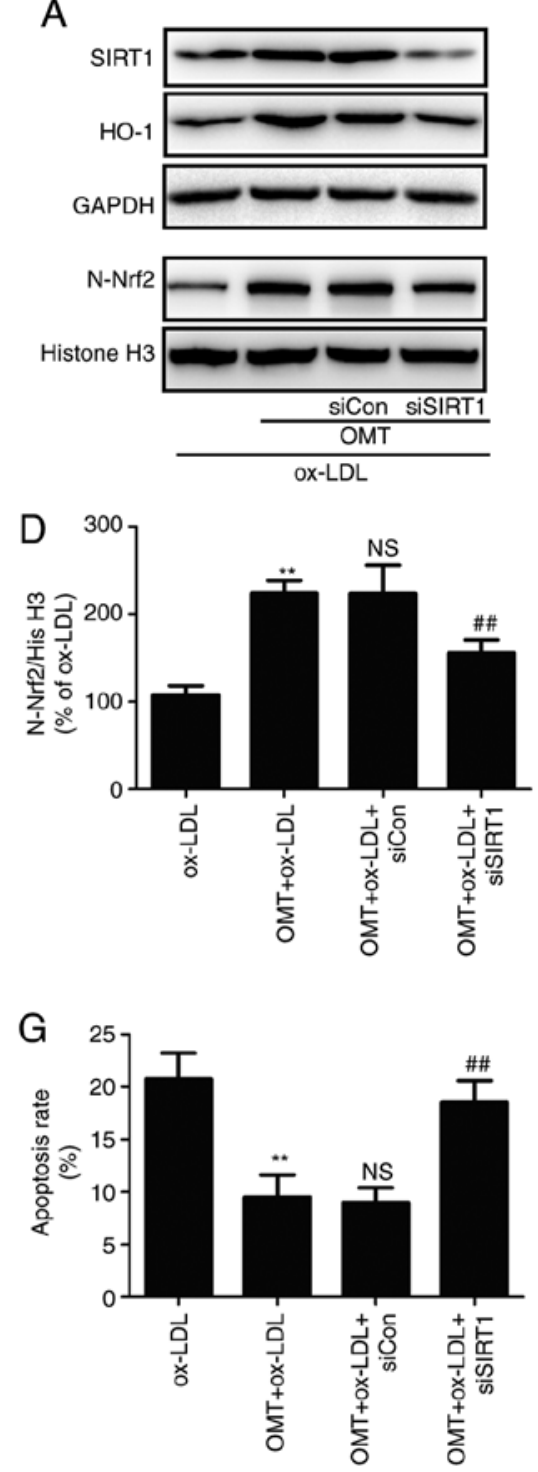
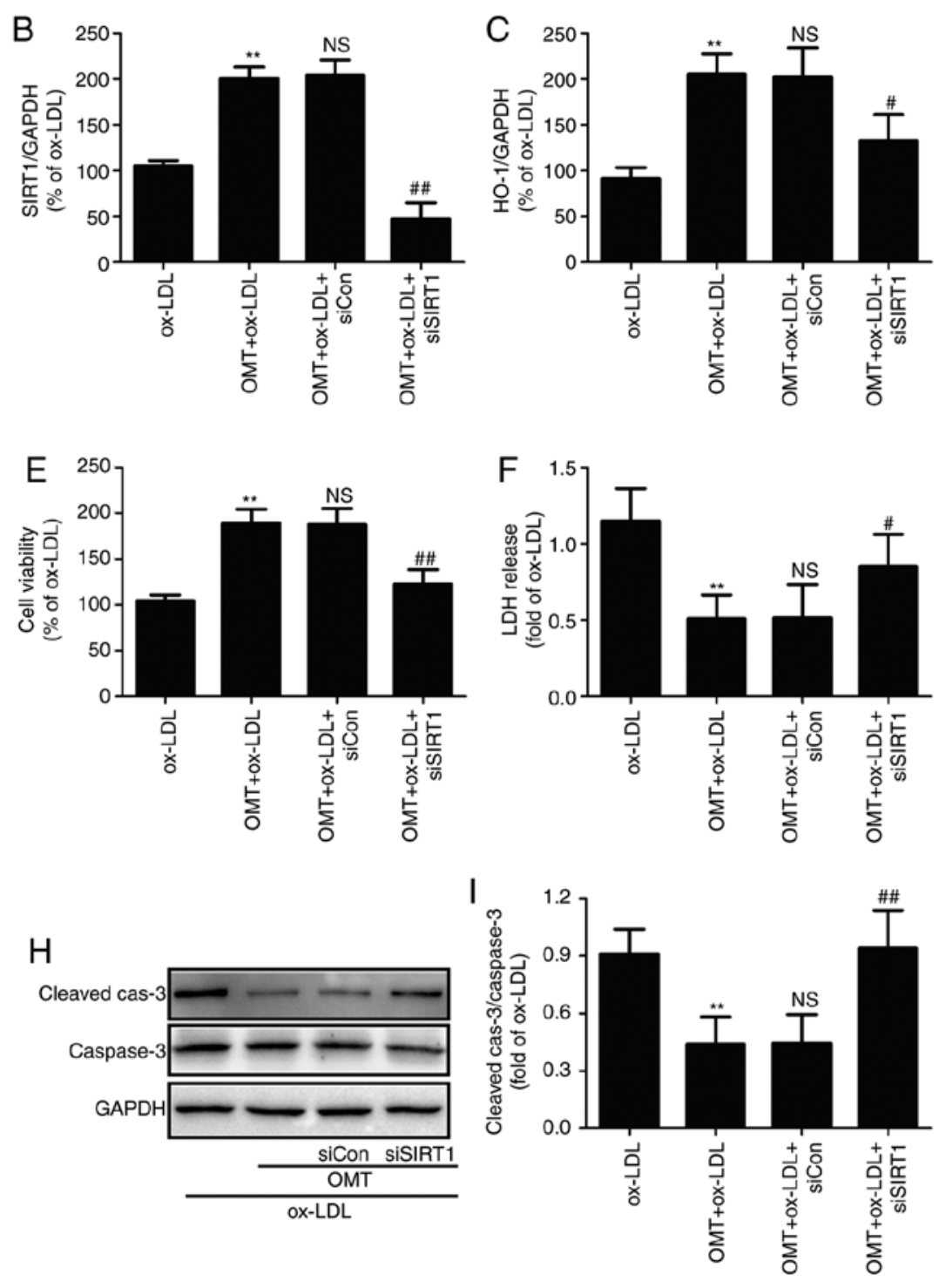

Figure 4. Effects of siSIRT1 transfection on the protective effects of oxymatrine against ox-LDL-induced injury in HUVECs. HUVECs transfected with siSIRT1 or siCon were treated with oxymatrine $(4 \mu \mathrm{M})$ for $2 \mathrm{~h}$ prior to treatment with ox-LDL (100 $\mu \mathrm{g} / \mathrm{ml})$ for $24 \mathrm{~h}$. (A) Western blot analysis for protein expression. Quantitative analysis for (B) SIRT1 and (C) HO-1 relative to GAPDH. (D) Quantitative analysis for N-Nrf2 relative to histone H3. Determination of (E) cell viability, (F) LDH release and (G) apoptosis rate. (H) Western blot analysis for cleaved caspase-3 and caspase-3 protein expression. (I) Quantitative analysis for cleaved caspase-3 relative to caspase-3. Data are expressed as the mean $\pm \mathrm{SD}, \mathrm{n}=3$. ${ }^{* *} \mathrm{P}<0.01$ vs. the ox-LDL group; ${ }^{\mathrm{NS}} \mathrm{P}>0.05$ vs. the OMT + ox-LDL group; ${ }^{\#} \mathrm{P}<0.05$ and ${ }^{\# \#} \mathrm{P}<0.01$ vs. the OMT + ox-LDL + siCon group. OMT, oxymatrine; ox-LDL, oxidized low-density lipoprotein; NS, no statistical significance; siSIRT1, siRNAs for SIRT1; siCon, siRNAs for negative control; HUVECs, human umbilical vein endothelial cells; LDH, lactate dehydrogenase; SIRT, sirtuin; Nrf2, nuclear factor-erythroid 2-related factor 2; C-Nrf2, cytoplasmic Nrf2; N-Nrf2, nuclear Nrf2; HO-1, heme oxygenase 1.

stress, and contributed to the inhibitory effect of oxymatrine on ox-LDL-induced NLRP3 inflammasome-mediated pyroptosis in HUVECs. These results indicated that oxymatrine may mitigate ox-LDL injury by inhibiting NLRP3 inflammasome-mediated pyroptosis via activating the Sirt1/Nrf2 signaling pathway, providing new insight into a functional role of oxymatrine in atherosclerosis.

Atherosclerosis is a multifactorial, chronic inflammatory disease that causes injury of endothelial cells, which are the first to be exposed to the effects of stimuli and physiological factors from their surroundings $(2,36)$. Oxymatrine, a natural bioactive compound, has been demonstrated to possess anti-inflammatory, antioxidant, antiviral, antifibrotic and cardioprotective properties (19,37). Recently, Zhang et al (19) revealed that oxymatrine may inhibit homocysteine-induced endothelial dysfunction, a hallmark of atherosclerosis, by attenuating autophagy-activated HUVEC apoptosis/death. Another study also demonstrated that oxymatrine prevented homocysteine-induced endothelial injury via attenuating mitochondrial-dependent apoptosis (18). Consistent with these studies, the present study also demonstrated that oxymatrine alleviated ox-LDL-induced cytotoxicity and apoptosis in HUVECs. Bcl-2 family proteins are involved in the mitochondria-dependent apoptosis pathway (38). The results of the present study further demonstrated that oxymatrine reversed the ox-LDL-induced increase in Bax expression and the decrease in Bcl-2 expression in HUVECs. Collectively, these results indicated that oxymatrine protected HUVECs against ox-LDL injury via inhibiting the mitochondria-dependent apoptosis pathway. 


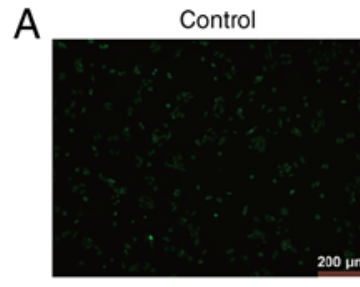

B
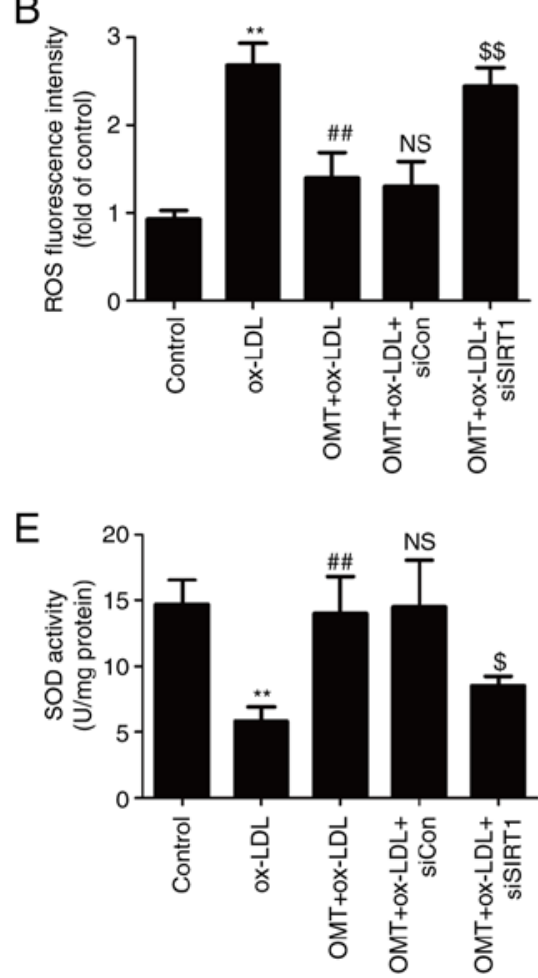

OX-LDL

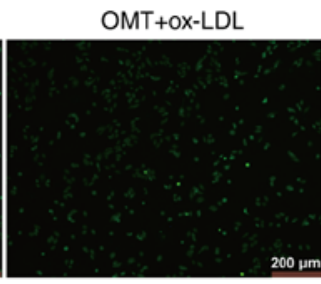

C

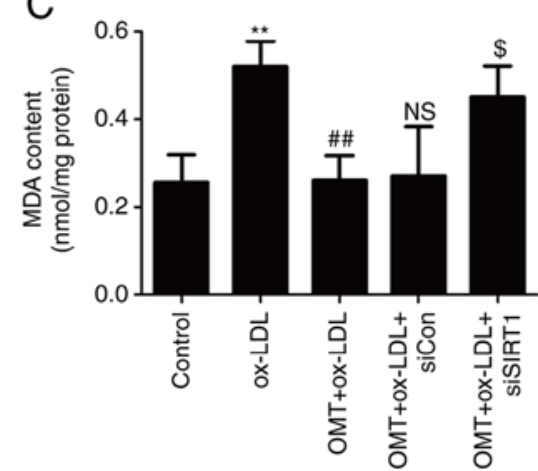

F

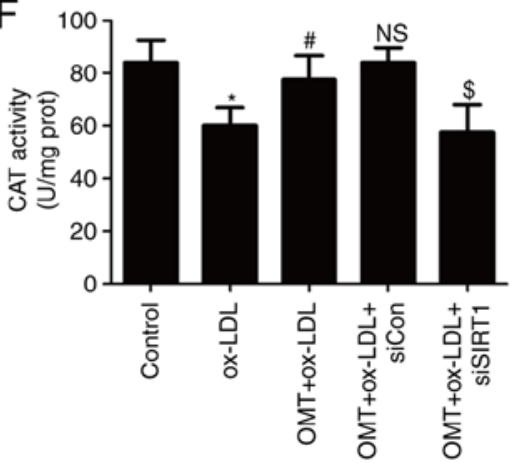

OMT+ox-LDL+siCon

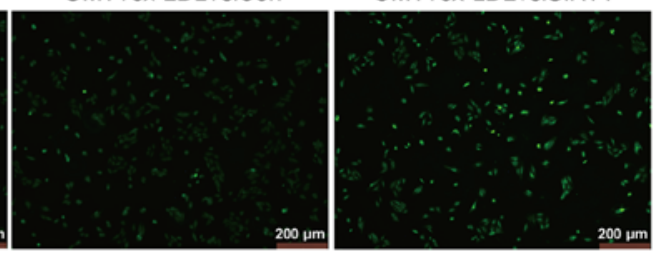

D
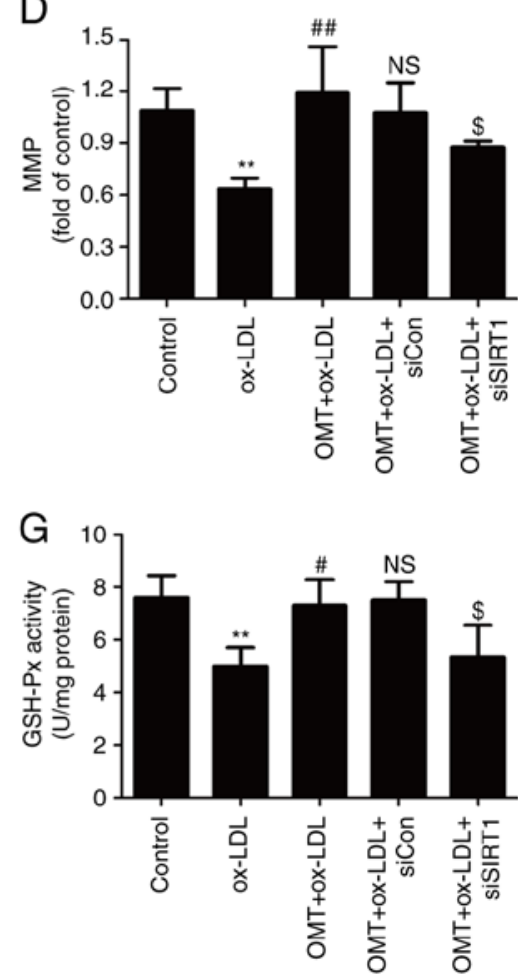

Figure 5. Effects of siSIRT transfection on the inhibitory effect of oxymatrine on oxidative stress in ox-LDL-treated HUVECs. HUVECs transfected with siSIRT1 or siCon were treated with oxymatrine $(4 \mu \mathrm{M})$ for $2 \mathrm{~h}$ prior to treatment with ox-LDL (100 $\mu \mathrm{g} / \mathrm{ml})$ for $24 \mathrm{~h}$. (A) DCFH-DA staining for ROS. Scale bar, $200 \mu \mathrm{m}$. (B) Quantitative analysis for ROS generation by flow cytometry. (C) Cell MDA assay kit for measuring MDA content. (D) JC-1 kit for measuring mitochondrial membrane potential. (E) SOD assay kit for measuring SOD activity. (F) CAT assay kit for measuring CAT activity. (G) GSH-Px assay kit for measuring GSH-Px activity. Data are expressed as the mean $\pm \mathrm{SD}, \mathrm{n}=3$. ${ }^{*} \mathrm{P}<0.05$ and ${ }^{* *} \mathrm{P}<0.01$ vs. the control group; ${ }^{\#} \mathrm{P}<0.05$ and ${ }^{\# \#} \mathrm{P}<0.01$ vs. the ox-LDL group; ${ }^{\mathrm{N}} \mathrm{P}>0.05$ vs. the OMT + ox-LDL group; ${ }^{\$} \mathrm{P}<0.05$ and ${ }^{\$ \$} \mathrm{P}<0.01$ vs. the OMT + ox-LDL + siCon group. OMT, oxymatrine; ox-LDL, oxidized low-density lipoprotein; NS, no statistical significance; siSIRT1, siRNAs for SIRT1; siCon, siRNAs for negative control; HUVECs, human umbilical vein endothelial cells; SIRT, sirtuin; MDA, malondialdehyde; SOD, superoxide dismutase; CAT, catalase; GSH-Px, glutathione peroxidase; ROS, reactive oxygen species; DCFH-DA, 2',7'-dichlorofluorescein diacetate.

Inflammation is an important driver of atherosclerosis, and therapeutic targeting of inflammatory pathways is suggested to ameliorate atherosclerosis $(39,40)$. NLRP3 inflammasomes are intracellular complexes involved in the inflammatory response that increases the levels of mature IL-1 $\beta$ and IL-18 and initiates pyroptosis via cleaving procaspase-1, playing a decisive role in atherosclerosis $(3,41)$. A number of studies have revealed that oxymatrine plays a markedly beneficial role in multiple diseases due to its profound anti-inflammatory effects $(37,42,43)$. However, to the best of our knowledge, the impact of oxymatrine on NLRP3 inflammasomes and pyroptosis has not been explored in atherosclerosis. The present study demonstrated that oxymatrine significantly inhibited the ox-LDL-induced increase in the expression of NLRP3 and ASC, as well as the expression of cleaved caspase-1, IL-1 $\beta$ and IL-18 in HUVECs, indicating that oxymatrine attenuated NLRP3 inflammasome activation-mediated pyroptosis under conditions of ox-LDL stimulation. In addition, the results further revealed that inhibition of NLRP3 inflammasome activation-mediated pyroptosis induced by siNLRP3 transfection inhibited the protective effects of oxymatrine against ox-LDL-induced HUVEC injury. Collectively, these results indicated that oxymatrine protected HUVECs against ox-LD-induced injury via inhibiting NLRP3 inflammasome-mediated pyroptosis.

SIRT1 was previously revealed to be involved in multiple cellular processes, and exerts marked protective effects against endothelial cell injury, vascular aging, inflammation and atherosclerotic plaque development $(22,23)$. It was recently reported that oxymatrine plays a beneficial role in ischemic stroke (29) and non-alcoholic fatty liver disease (29) via promoting SRT1 signaling. However, the effect of oxymatrine on the SIRT1 signaling pathway in atherosclerosis remains unknown. In the present study, the results revealed that oxymatrine pretreatment markedly increased the expression of SIRT1 in ox-LDL-treated HUVECs. Nrf2, a key antioxidant sensor for cellular defense mechanisms, is considered as an important downstream target of SIRT1 and increases resistance to oxidative damage; it is also closely associated with atherosclerosis 
A

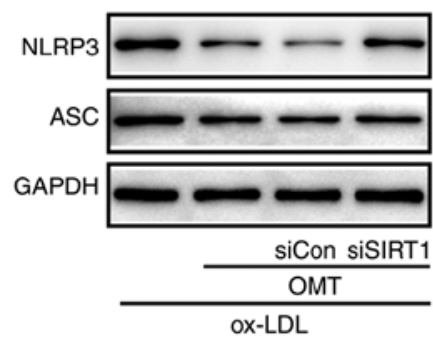

D

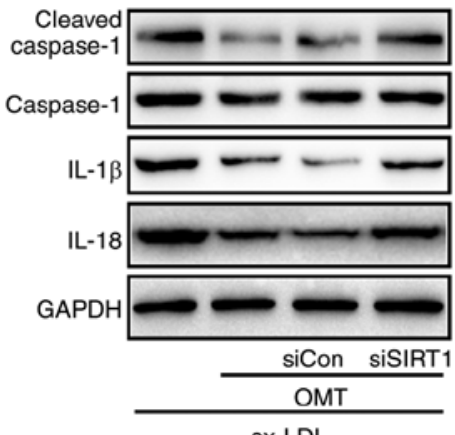

B

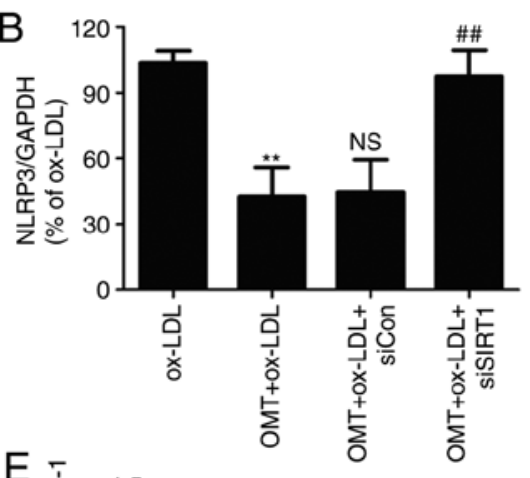

C

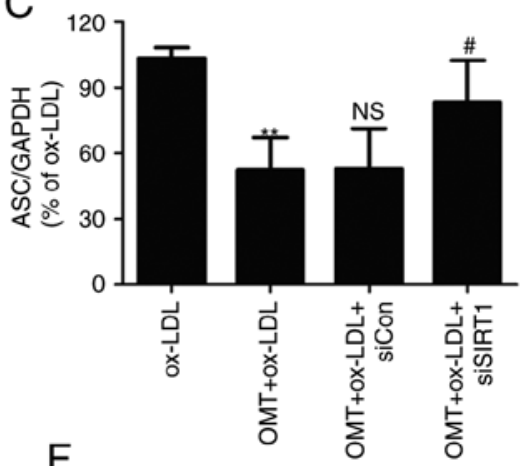

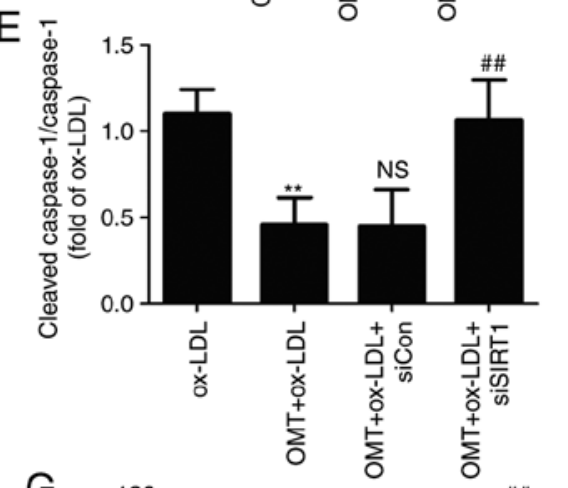
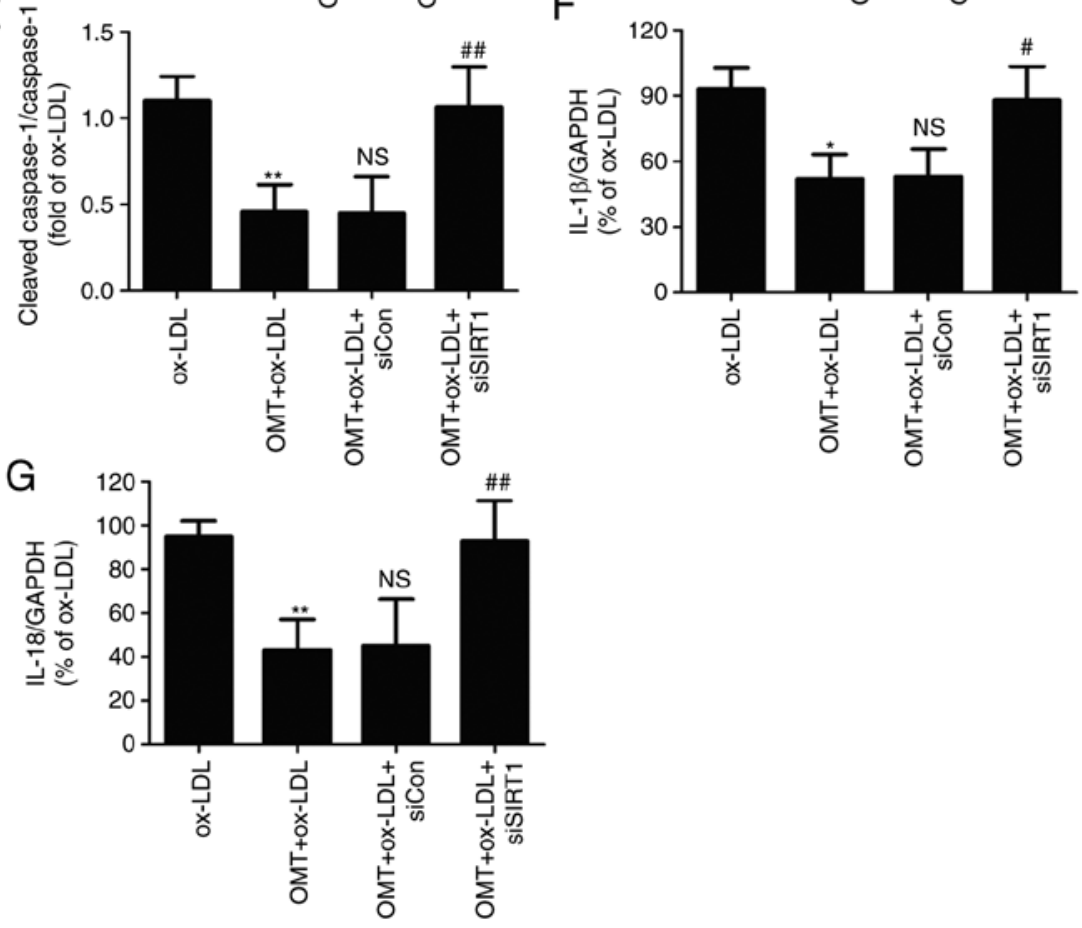

Figure 6. Effects of siSIRT transfection on the inhibitory effect of oxymatrine against NLRP3 inflammasome-mediated pyroptosis in ox-LDL-treated HUVECs. HUVECs transfected with siSIRT1 or siCon were treated with oxymatrine $(4 \mu \mathrm{M})$ for $2 \mathrm{~h}$ prior to treatment with ox-LDL (100 $\mu \mathrm{g} / \mathrm{ml})$ for $24 \mathrm{~h}$. (A) Western blot analysis for protein expression. Quantitative analysis for (B) NLRP3 and (C) ASC relative to GAPDH. (D) Western blot analysis for protein expression. Quantitative analysis for (E) cleaved caspase-1/caspase-1, (F) IL-1 $\beta$ and $(\mathrm{G}) \mathrm{IL}-18$ relative to GAPDH. Data are expressed as the mean $\pm \mathrm{SD}$, $\mathrm{n}=3$. ${ }^{* *} \mathrm{P}<0.01$ vs. the ox-LDL group; ${ }^{\mathrm{NS}} \mathrm{P}>0.05$ vs. the OMT + ox-LDL group; ${ }^{\#} \mathrm{P}<0.05$ and ${ }^{\# \#} \mathrm{P}<0.01$ vs. the OMT + ox-LDL + siCon group. OMT, oxymatrine; ox-LDL, oxidized low-density lipoprotein; NS, no statistical significance; siSIRT1, siRNAs for SIRT1; siCon, siRNAs for negative control; HUVECs, human umbilical vein endothelial cells; NLRP3, NLR family pyrin domain containing 3; SIRT, sirtuin; ASC, apoptosis-associated speck-like protein containing a C-terminal caspase recruitment domain.

development (25). Under normal conditions, Nrf2 binds to its negative regulator, Kelch-like ECH-associated protein 1 (Keap1), which leads to its degradation via ubiquitination. In response to oxidative stress, Nrf2 is dissociated from Keap1 and translocates to the nucleus, thereby activating antioxidant responses by upregulating the expression of several antioxidant enzymes, particularly HO-1 $(44,45)$. Consistent with these previous findings, the present study demonstrated that ox-LDL increased the expression of $\mathrm{C}-\mathrm{Nrf} 2$, decreased the expression of N-Nrf2, and enhanced the expression of HO-1, which were also attenuated by oxymatrine. These results indicated that oxymatrine activated the SIRT1/Nrf2 signaling pathway in ox-LDL-treated HUVECs. Furthermore, the present study demonstrated that inhibition of SIRT1/Nrf2 signaling induced by siSIRT1 transfection mitigated the protective effects of oxymatrine against ox-LDL injury in HUVECs. Collectively, these results indicated that the SIRT1/Nrf2 signaling pathway mediated the cardioprotective function of oxymatrine in atherosclerosis.

An increasing number of studies have revealed that the mechanisms underlying the protective effects of oxymatrine are mainly associated with its anti-inflammatory and antioxidant properties $(13,37,42)$. Similarly, the present results demonstrated that oxymatrine markedly attenuated ox-LDL-induced oxidative stress, as evidenced by the decrease in ROS generation and MDA content, and the increase in MMP and the activities of the antioxidant enzymes SOD, CAT and GSH-Px in HUVECs. Previous studies have reported that SIRT1/Nrf2 signaling dysregulation is involved in the ability to scavenge oxygen free radicals through the action of SOD, CAT and GSH-Px $(46,47)$. Consistent with these studies, the present results further revealed that the inhibition of SIRT1/Nrf2 signaling pathway through siSIRT1 transfection eliminated the antioxidant properties of oxymatrine under conditions of 
ox-LDL stimulation. These results indicated that oxymatrine attenuated ox-LDL-induced injury by attenuating oxidative stress via promoting SIRT1/Nrf2 signaling in HUVECs.

An increasing number of studies have reported that activating SIRT1/Nrf2 signaling may reduce NLRP3 inflammasome activation (48) and pyroptosis (49). However, the role of the SIRT1/Nrf2 signaling pathway in the inhibitory effect of oxymatrine on NLRP3 inflammasome-mediated pyroptosis has not been reported to date. In the present study, the results demonstrated that siSIRT1 also abolished the inhibitory effect of oxymatrine on NLRP3 inflammasome-mediated pyroptosis, which was similar to the findings of previous studies reporting that NLRP3 inflammasome activation was associated with Nrf2 and SIRT1 activation and can be reversed by Nrf2 siRNA and SIRT1 inhibitor treatment $(48,50)$. These results indicated that oxymatrine reduced NLRP3 inflammasome-mediated pyroptosis via enhancing SIRT1/Nrf2 signaling in ox-LDL-treated HUVECs.

Some shortcomings must be acknowledged in this study. Firstly, no specific immunofluorescence markers for pyroptosis in HUVECs were tested. Secondly, our experiments are limited to HUVECs, other cells or an in vivo experimental model have not yet been considered.

Collectively, the findings of the present study demonstrated that oxymatrine protected HUVECs against ox-LDL-induced injury by inhibiting NLRP3 inflammasome-mediated pyroptosis via activation of the SIRT1/Nrf2 signaling pathway. The results indicated that oxymatrine may serve as a potential therapeutic candidate for the treatment of atherosclerosis, and suggested a new strategy for targeting SIRT1/Nrf2 signaling and NLRP3 inflammasome-mediated pyroptosis.

\section{Acknowledgements}

Not applicable.

\section{Funding}

The present study was supported by the Hunan Provincial Natural Science Foundation of China (grant nos. 2018JJ3465 and 2019JJ50551).

\section{Availability of data and materials}

The datasets generated and/or analyzed in the present study are available from the corresponding author upon reasonable request.

\section{Authors' contributions}

$\mathrm{XJ}$ and BW conceived, designed the experiments, and confirmed the authenticity of the raw data. XJ, WF and JZ performed the experiments and analyzed the data. NS and YY collected and analyzed the data. XJ, YY and BW wrote and revised the manuscript. All the authors have read and approved the final manuscript.

\section{Ethics approval and consent to participate}

Not applicable.

\section{Patient consent for publication}

Not applicable.

\section{Competing interests}

The authors declare that they have no competing interests.

\section{References}

1. Kobiyama K and Ley K: Atherosclerosis. Circ Res 123: 1118-1120, 2018.

2. Libby P, Bornfeldt KE and Tall AR: Atherosclerosis: Successes, surprises, and future challenges. Circ Res 118: 531-534, 2016.

3. Baldrighi M, Mallat Z and Li X: NLRP3 inflammasome pathways in atherosclerosis. Atherosclerosis 267: 127-138, 2017.

4. Paramel Varghese G, Folkersen L, Strawbridge RJ, Halvorsen B, Yndestad A, Ranheim T, Krohg-Sørensen K, Skjelland M, Espevik T, Aukrust P, et al: NLRP3 inflammasome expression and activation in human atherosclerosis. J Am Heart Assoc 5: e003031, 2016

5. Jiang C, Jiang L, Li Q, Liu X, Zhang T, Dong L, Liu T, Liu L, Hu G, Sun X and Jiang L: Acrolein induces NLRP3 inflammasome-mediated pyroptosis and suppresses migration via ROS-dependent autophagy in vascular endothelial cells. Toxicology 410: 26-40, 2018.

6. Qiu Z, Lei S, Zhao B, Wu Y, Su W, Liu M, Meng Q, Zhou B, Leng Y and Xia ZY: NLRP3 inflammasome activation-mediated pyroptosis aggravates myocardial ischemia/reperfusion injury in diabetic rats. Oxid Med Cell Longev 2017: 9743280, 2017.

7. Sutterwala FS, Haasken S and Cassel SL: Mechanism of NLRP3 inflammasome activation. Ann NY Acad Sci 1319: 82-95, 2014.

8. Jia C, Zhang J, Chen H, Zhuge Y, Chen H, Qian F, Zhou K, Niu C, Wang F, Qiu H, et al: Endothelial cell pyroptosis plays an important role in Kawasaki disease via HMGB1/RAGE/cathespin B signaling pathway and NLRP3 inflammasome activation. Cell Death Dis 10: 778, 2019.

9. LiP,Zhong X,LiJ,LiuH,MaX,HeRandZhao Y:MicroRNA-30c-5p inhibits NLRP3 inflammasome-mediated endothelial cell pyroptosis through FOXO3 down-regulation in atherosclerosis. Biochem Biophys Res Commun 503: 2833-2840, 2018.

10. Zahid A, Li B, Kombe AJK, Jin T and Tao J: Pharmacological Inhibitors of the NLRP3 Inflammasome. Front Immunol 10: 2538, 2019.

11. Zhang L, Yuan M, Zhang L, Wu B and Sun X: Adiponectin alleviates NLRP3-inflammasome-mediated pyroptosis of aortic endothelial cells by inhibiting FoxO4 in arteriosclerosis. Biochem Biophys Res Commun 514: 266-272, 2019.

12. Liu Y, Wang H, Liu N, Du J, Lan X, Qi X, Zhuang C, Sun T, $\mathrm{Li} \mathrm{Y}$ and $\mathrm{Yu}$ J: Oxymatrine protects neonatal rat against hypoxic-ischemic brain damage via PI3K/Akt/GSK3 $\beta$ pathway. Life Sci 254: 116444, 2020.

13. Liang J, Chang B, Huang M, Huang W, Ma W, Liu Y, Tai W, Long Y and $\mathrm{Lu}$ Y: Oxymatrine prevents synovial inflammation and migration via blocking NF- $\mathrm{KB}$ activation in rheumatoid fibroblast-like synoviocytes. Int Immunopharmacol 55: 105-111, 2018.

14. Zhang YY, Yi M and Huang YP: Oxymatrine ameliorates doxorubicin-induced cardiotoxicity in rats. Cell Physiol Biochem 43: 626-635, 2017.

15. Hu ST, Tang Y, Shen YF, Ao HH, Bai J, Wang YL and Yang YJ: Protective effect of oxymatrine on chronic rat heart failure. J Physiol Sci 61: 363-372, 2011.

16. Yang Y, Chen S, Tao L, Gan S, Luo H, Xu Y and Shen X: Inhibitory effects of oxymatrine on transdifferentiation of neonatal rat cardiac fibroblasts to myofibroblasts induced by aldosterone via Keap1/Nrf2 signaling pathways in vitro. Med Sci Monit 25: 5375-5388, 2019.

17. Huang XY and Chen CX: Effect of oxymatrine, the active component from Radix Sophorae flavescentis (Kushen), on ventricular remodeling in spontaneously hypertensive rats. Phytomedicine 20: 202-212, 2013.

18. Wu B, Yue H, Zhou GH, Zhu YY, Wu TH, Wen JF, Cho KW and Jin SN: Protective effects of oxymatrine on homocysteine-induced endothelial injury: Involvement of mitochondria-dependent apoptosis and Akt-eNOS-NO signaling pathways. Eur J Pharmacol 864: 172717, 2019. 
19. Zhang Y, Zhang Y, Tang J, Zhao S, Li C, Huang YP and Yi M Oxymatrine inhibits homocysteine-mediated autophagy via $\mathrm{MIF} / \mathrm{mTOR}$ signaling in human umbilical vein endothelial cells. Cell Physiol Biochem 45: 1893-1903, 2018.

20. Khatana C, Saini NK, Chakrabarti S, Saini V, Sharma A Saini RV and Saini AK: Mechanistic insights into the oxidized low-density lipoprotein-induced atherosclerosis. Oxid Med Cell Longev 2020: 5245308, 2020.

21. Kitada M, Ogura Y and Koya D: The protective role of Sirt1 in vascular tissue: Its relationship to vascular aging and atherosclerosis. Aging (Albany NY) 8: 2290-2307, 2016.

22. Zhang MJ, Zhou Y, Chen L, Wang X, Long CY, Pi Y, Gao CY, Li JC and Zhang LL: SIRT1 improves VSMC functions in atherosclerosis. Prog Biophys Mol Biol 121: 11-15, 2016.

23. Stein $\mathrm{S}$ and MatterCM: Protective roles of SIRT1 in atherosclerosis. Cell Cycle 10: 640-647, 2011

24. Lazaro I, Lopez-Sanz L, Bernal S, Oguiza A, Recio C, Melgar A, Jimenez-Castilla L, Egido J, Madrigal-Matute J and Gomez-Guerrero C: Nrf2 activation provides atheroprotection in diabetic mice through concerted upregulation of antioxidant, anti-inflammatory, and autophagy mechanisms. Front Pharmacol 9: 819, 2018.

25. Mimura J and Itoh K: Role of Nrf2 in the pathogenesis of atherosclerosis. Free Radic Biol Med 88: 221-232, 2015.

26. Gao Z, Sui J, Fan R, Qu W, Dong X and Sun D: Emodin protects against acute pancreatitis-associated lung injury by inhibiting NLPR3 inflammasome activation via $\mathrm{Nrf} 2 / \mathrm{HO}-1$ signaling. Drug Des Devel Ther 14: 1971-1982, 2020.

27. Hou Y, Wang Y, He Q, Li L, Xie H, Zhao Y and Zhao J: Nrf2 inhibits NLRP3 inflammasome activation through regulating Trx1/TXNIP complex in cerebral ischemia reperfusion injury. Behav Brain Res 336: 32-39, 2018

28. Xu H, Chen GF, Ma YS, Zhang HW, Zhou Y, Liu GH, Chen DY, Ping J, Liu YH, Mou X and Fu D: Hepatic proteomic changes and Sirtl/AMPK signaling activation by oxymatrine treatment in rats with non-alcoholic steatosis. Front Pharmacol 11: 216, 2020

29. Zhou S, Qiao B, Chu X and Kong Q: Oxymatrine attenuates cognitive deficits through SIRT1-mediated autophagy in ischemic stroke. J Neuroimmunol 323: 136-142, 2018.

30. Zhu Z, Li J and Zhang X: Salidroside protects against ox-LDL-induced endothelial injury by enhancing autophagy mediated by SIRT1-FoxO1 pathway. BMC Complement Altern Med 19: 111, 2019.

31. Edlich F: BCL-2 proteins and apoptosis: Recent insights and unknowns. Biochem Biophys Res Commun 500: 26-34, 2018.

32. Grebe A, Hoss F and Latz E: NLRP3 inflammasome and the IL-1 pathway in atherosclerosis. Circ Res 122: 1722-1740, 2018.

33. Esteban-Fernándezde Ávila B,Ramírez-Herrera DE,CampuzanoS, Angsantikul P, Zhang L and Wang J: Nanomotor-enabled pH-responsive intracellular delivery of caspase-3: Toward rapid cell apoptosis. CS Nano 11: 5367-5374, 2017.

34. Dubois-Deruy E, Peugnet V, Turkieh A and Pinet F: Oxidative stress in cardiovascular diseases. Antioxidants (Basel) 9: 864, 2020.

35. Li Y, Wang P, Yang X, Wang W, Zhang J, He Y, Zhang W, Jing T, Wang B and Lin R: SIRT1 inhibits inflammatory response partly through regulation of NLRP3 inflammasome in vascular endothelial cells. Mol Immunol 77: 148-156, 2016.
36. Falk E: Pathogenesis of atherosclerosis. J Am Coll Cardiol 47 (Suppl 8): C7-C12, 2006.

37. Lan X, Zhao J, Zhang Y, Chen Y, Liu Y and Xu F: Oxymatrine exerts organ- and tissue-protective effects by regulating inflammation, oxidative stress, apoptosis, and fibrosis: From bench to bedside. Pharmacol Res 151: 104541, 2020.

38. King KL and Cidlowski JA: Cell cycle regulation and apoptosis. Annu Rev Physiol 60: 601-617, 1998

39. Bäck M, Yurdagul A Jr, Tabas I, Öörni K and Kovanen PT: Inflammation and its resolution in atherosclerosis: Mediators and therapeutic opportunities. Nat Rev Cardiol 16: 389-406, 2019.

40. Nasonov EL and Popkova TV: Atherosclerosis: Perspectives of anti-inflammatory therapy. Ter Arkh 90: 4-12, 2018.

41. Hoseini Z, Sepahvand F, Rashidi B, Sahebkar A, Masoudifar A and Mirzaei H: NLRP3 inflammasome: Its regulation and involvement in atherosclerosis. J Cell Physiol 233: 2116-2132, 2018.

42. Dong P, Ji X, Han W and Han H: Oxymatrine exhibits anti-neuroinflammatory effects on $\mathrm{A} \beta_{1-42}$-induced primary microglia cells by inhibiting NF- $\kappa \mathrm{B}$ and MAPK signaling pathways. Int Immunopharmacol 74: 105686, 2019.

43. Jiang Y, Sang W, Wang C, Lu H, Zhang T, Wang Z, Liu Y, Xue B, Xue S, Cai Z, et al: Oxymatrine exerts protective effects on osteoarthritis via modulating chondrocyte homoeostasis and suppressing osteoclastogenesis. J Cell Mol Med 22: 3941-3954, 2018.

44. Bellezza I, Giambanco I, Minelli A and Donato R: Nrf2-Keap1 signaling in oxidative and reductive stress. Biochim Biophys Acta Mol Cell Res 1865: 721-733, 2018.

45. Chen QM and Maltagliati AJ: Nrf2 at the heart of oxidative stress and cardiac protection. Physiol Genomics 50: 77-97, 2018.

46. Chen XJ, Wu WJ, Zhou Q, Jie JP, Chen X, Wang F and Gong XH: Advanced glycation end-products induce oxidative stress through the Sirt1/Nrf2 axis by interacting with the receptor of AGEs under diabetic conditions. J Cell Biochem: Oct 15, 2018 (Epub ahead of print). doi: 10.1002/jcb.27524.

47. Zhang B, Zhai M, Li B, Liu Z, Li K, Jiang L, Zhang M, Yi W, Yang J, Yi D, et al: Honokiol ameliorates myocardial ischemia/reperfusion injury in type 1 diabetic rats by reducing oxidative stress and apoptosis through activating the SIRT1-Nrf2 signaling pathway. Oxid Med Cell Longev 2018: 3159801, 2018.

48. Arioz BI, Tastan B, Tarakcioglu E, Tufekci KU, Olcum M, Ersoy N, Bagriyanik A, Genc K and Genc S: Melatonin attenuates LPS-induced acute depressive-like behaviors and microglial NLRP3 inflammasome activation through the SIRT1/Nrf2 pathway. Front Immunol 10: 1511, 2019.

49. Yin Y, Wu X, Peng B, Zou H, Li S, Wang J and Cao J: Curcumin improves necrotising microscopic colitis and cell pyroptosis by activating SIRT1/NRF2 and inhibiting the TLR4 signalling pathway in newborn rats. Innate Immun 26: 609-617, 2020.

50. Zhang S, Jiang L, Che F, Lu Y, Xie Z and Wang H: Arctigenin attenuates ischemic stroke via SIRT1-dependent inhibition of NLRP3 inflammasome. Biochem Biophys Res Commun 493: 821-826, 2017.

This work is licensed under a Creative Commons Attribution-NonCommercial-NoDerivatives 4.0 International (CC BY-NC-ND 4.0) License. 